\title{
Avaliação da utilização das cinzas da escuma gerada em Reator Anaeróbico de Manta de Lodo e Fluxo Ascendente como pigmento inorgânico de coloração alaranjada
}

\author{
Evaluation of the use of ashes of scum generated from the \\ Upflow Anaerobic Sludge Blanket reactor as orange inorganic pigment \\ Tanna Elyn Rodrigues Fiuza', Eder Carlos Ferreira de Souza², Sandra Regina Masetto Antunes ${ }^{3}$, \\ Wilson Costa ${ }^{4}$, Maria Elena Payret Arrúa ${ }^{5}$, Augusto Celso Antunes ${ }^{6}$
}

\begin{abstract}
RESUMO
A escuma é um resíduo gerado durante o tratamento anaeróbico de efluentes domésticos que necessita de periódica remoção para não comprometer a eficiência do processo. O presente trabalho teve por objetivo utilizar as cinzas da escuma gerada em Reator Anaeróbico de Manta de Lodo e Fluxo Ascendente (RALF) como pigmentos inorgânicos. Amostras de escumas coletadas em diferentes meses foram submetidas a tratamento térmico a $900^{\circ} \mathrm{C}$ por $1 \mathrm{~h}$ para remoção da matéria orgânica. As cinzas obtidas foram desaglomeradas em peneira e submetidas às análises química (Fluorescência de raios $X$ - FRX), mineralógica (Difração de raios X - DRX), morfológica (Microscopia Eletrônica de Varredura por Efeito de Campo - FE-MEV) e de coloração (espectroscopia de reflectância difusa na região do Ultravioleta-Visível - UV-Vis). As amostras apresentaram composição majoritária de elementos comuns no ambiente, sendo as fases principais identificadas o quartzo (dióxido de silício - $\mathrm{SiO}_{2}$ ), a anidrita (sulfato de cálcio - $\mathrm{CaSO}_{4}$ ) e a hematita (óxido de ferro - $\mathrm{Fe}_{2} \mathrm{O}_{3}$ ). A granulometria das amostras é adequada para a aplicação como pigmento cerâmico; a coloração obtida foi alaranjada intensa e boas propriedades foram identificadas, como boa opacidade, omitindo a coloração do suporte cerâmico, além de não existirem defeitos superficiais que indicassem matéria orgânica residual ou incompatibilidade com o esmalte utilizado. Sendo assim, as cinzas da escuma gerada em RALF apresentaram características interessantes para a aplicação como pigmentos cerâmicos.
\end{abstract}

Palavras-chave: resíduo; esgoto; escuma; pigmento inorgânico.

\begin{abstract}
Scum is a residue generated during the anaerobic treatment of domestic effluents and requires periodical removal in order not to harm the process efficacy. This study aimed to use the scum ashes generated from Upflow Anaerobic Sludge Blanket (UASB) reactor as inorganic pigments. The scum samples collected in different months were thermally treated at $900^{\circ} \mathrm{C}$ for one hour for removal of organic matter. These ashes were dispersed and submitted to chemical (X-ray Fluorescence - XRF), mineralogical (X-ray Diffraction - XRD), morphological (Field Emission Scanning Electron Microscopy - FE-SEM) and color (Ultraviolet-Visible Diffuse Reflectance Spectroscopy - UV-Vis DRS) analyses. The samples were composed mainly by elements commonly found in the environment. The main crystalline phases are quartz (silicon dioxide - $\mathrm{SiO}_{2}$ ), anhydrite (calcium sulfate $-\mathrm{CaSO}_{4}$ ) and hematite (iron oxide $-\mathrm{Fe}_{2} \mathrm{O}_{3}$ ). Also, these particles presented suitable size for application as ceramic pigment and the color obtained was intense orange. When applied as ceramic pigment at the decorative fire temperature, the enameled piece presented intense color and the pigment had good opacity, hiding the ceramic support color, besides, they presented no surface deformations, which indicates the pigments are compatible with enamel. Thus, scum ashes generated in UASB reactor presented interesting characteristics to be applied as ceramic pigments.
\end{abstract}

Keywords: residue; sewage; scum; inorganic pigment.

\section{$\square$}

'Doutoranda em Química pela Universidade Estadual de Campinas (UNICAMP) - Campinas (SP), Brasil.

2Doutor em Química pelo Instituto de Química da Universidade Estadual Paulista Júlio de Mesquita Filho (UNESP) - Araraquara (SP), Brasil. Professor do Departamento de Química da Universidade Estadual de Ponta Grossa (UEPG) - Ponta Grossa (PR), Brasil.

${ }^{3}$ Doutora em Química pela Universidade Federal de São Carlos (UFSCar) - São Carlos (SP), Brasil. Professora do Departamento de Química da Universidade Estadual de Ponta Grossa (UEPG) - Ponta Grossa (PR), Brasil.

${ }^{4}$ Doutor em Química Analítica pelo Instituto de Química da Universidade Estadual Paulista Júlio de Mesquita Filho (UNESP) - Araraquara (SP), Brasil. Professor do Departamento de Química da Universidade Estadual de Ponta Grossa (UEPG) - Ponta Grossa (PR), Brasil.

${ }^{5}$ Doutora em Química Orgânica pelo Instituto de Química da Universidade de São Paulo (USP) - São Paulo (SP), Brasil. Professora do Departamento de Química da Universidade Estadual de Ponta Grossa (UEPG) - Ponta Grossa (PR), Brasil.

${ }^{6}$ Doutor em Química pela Universidade Federal de São Carlos (UFSCar) - São Carlos (SP), Brasil. Professor do Departamento de Química da Universidade Estadual de Ponta Grossa (UEPG) - Ponta Grossa (PR), Brasil.

Endereço para correspondência: Carlos Ferreira de Souza - Universidade Estadual de Ponta Grossa - Departamento de Química - Avenida Gal. Carlos Cavalcanti, 4.748 Uvaranas - 84030-900 - Ponta Grossa (PR), Brasil - E-mail: ecfsouza@uepg.br

Recebido: 28/01/16 - Aceito: 24/10/16 - Reg. ABES: 158707 


\section{INTRODUÇÃO}

Os pigmentos inorgânicos são utilizados no cotidiano das civilizações desde a pré-história e, atualmente, são empregados em diversos ramos da indústria, como em cerâmicas, plásticos, tintas e borrachas (BARNETT; MILLER; PEARCE, 2006; GEORGE et al., 2008). No caso das indústrias de cerâmicas, esse tipo de pigmento é amplamente utilizado em revestimentos decorativos e também na proteção de porcelanas, corpos cerâmicos e vidrados (JANSEN \& LETSCHERT, 2000; BONDIOLI et al., 2004). Para tanto, os pigmentos são dispersos em fritas ou esmaltes e aplicados sobre uma superfície, sendo, em seguida, tratados termicamente (HOSSEINI-ZORI \& TAHERINASSAJ, 2011). Um problema relacionado aos pigmentos inorgânicos de uso comum é a presença de elementos tóxicos, como cádmio $(\mathrm{Cd})$, cromo $(\mathrm{Cr})$, chumbo $(\mathrm{Pb})$, entre outros. Por isso, são desejáveis novos pigmentos termicamente mais estáveis, com colorações mais intensas e com menor toxicidade ao ambiente e ao ser humano. Como exemplo dos novos pigmentos que estão sendo desenvolvidos, tem-se o uso de íons $\mathrm{Fe}^{3+}$ em sistemas com terras raras, que apresentam colorações industrialmente interessantes e com menor toxicidade se comparados aos elementos supramencionados (OLEGÁRIO et al., 2013; 2014; MARANHA et al., 2016). Entretanto, o custo mais elevado dos pigmentos sintéticos está associado à pureza dos reagentes utilizados, o que eleva o preço final dos produtos produzidos com tais colorantes para o consumidor. Desse modo, estudos que envolvem a aplicação de resíduos de processos industriais na síntese ou na aplicação direta como pigmentos ganham cada vez mais destaque (COSTA et al., 2008). A grande maioria das pesquisas reportadas na literatura envolve a utilização de lodos gerados em tratamento de resíduos industriais, que possuem elevados teores de elementos como ferro $(\mathrm{Fe})$, níquel $(\mathrm{Ni})$, cromo $(\mathrm{Cr})$, manganês $(\mathrm{Mn})$ e zinco (Zn) (COSTA et al., 2008; MARCELLO et al., 2008; HAJJAJI et al., 2011). Outros utilizam cinzas de resíduos de agricultura, como cinzas de cascas de arroz, que são ricas em silício (Si) (BONDIOLI et al., 2007; HABEEB \& MAHMUD, 2010).

No tratamento de esgotos municipais em reatores anaeróbicos existe a geração periódica de resíduos sólidos, como o lodo e a escuma. O lodo, quando perde sua vida útil, é removido dos reatores, desidratado em leitos de secagem e tratado para remoção de patógenos. Esse resíduo tem sido muito estudado na incorporação em cerâmicas, visando alterar suas propriedades e obter materiais com bom desempenho, além de imobilizar a fração inorgânica na matriz, reduzindo o potencial de contaminação (ANDREOLI, 2006; SAMAE POMERODE, 2015; SANEPAR, 2015; ZHANG et al., 2015). Entretanto, na agricultura, é possível constatar o uso cada vez mais crescente do lodo, após os processos de desidratação, estabilização, desinfecção e análise de teor de elementos tóxicos, sendo então denominados "biossólidos". Nos EUA e em parte da Europa, em torno de $60 \%$ desses lodos são utilizados como biossólidos na agricultura (MARGUÍ et al., 2016). No Brasil, a atividade ainda não é tão expressiva, mas existem relatos na literatura de que a prática melhora a fertilidade do solo, sendo testada em culturas como o milho e o eucalipto (POGGIANI \& MULLER, 2005; TRANNIN; SIQUEIRA; MOREIRA, 2008). Os biossólidos provenientes do tratamento de esgotos são conhecidos por alterar as propriedades físicas do solo, em especial no que diz respeito à porosidade, à capacidade de retenção de água e ao fato de conterem micro e macronutrientes (MELO; MARQUES; MELO, 2001).

A escuma, por sua vez, é um resíduo sólido gerado no tratamento de esgotos domésticos que possui, além do lodo que se solta do fundo do reator pelo fluxo de entrada do esgoto, óleos, graxas e resíduos grosseiros, como pedaços de plástico, que não são retidos nas etapas de pré-tratamento (CHERNICHARO et al., 2015). Sua remoção é necessária, pois dificulta a saída dos gases gerados no processo de degradação da matéria orgânica, além de ocupar volume útil do reator (CHERNICHARO et al., 2015). A escuma, mesmo passando pelas etapas de desidratação e desinfecção, não é recomendada para aplicação na agricultura por conter óleos e gorduras, pelo risco de impermeabilização do solo (RANKIN \& SCHLENZ, 1947; CHERNICHARO, 2010; METCALF \& EDDY INC. et al., 2003). Com isso, a escuma é um passivo ambiental, destinado a aterros, o que gera custos de transporte e disposição. Com a necessidade de ampliação dos sistemas de tratamento de esgoto, é inevitável o aumento da geração de escuma, o que se torna um problema ambiental e econômico. Por isso, buscar métodos para utilização da escuma é essencial para viabilizar a ampliação de sistemas de tratamento de esgotos. Na literatura, é relatada a caracterização da fração oleosa da escuma visando à produção de biodiesel, quando essa apresenta elevado teor de óleos e graxas (OLIVEIRA et al., 2014; DI BITONTO et al., 2016). Outro uso mencionado é a desidratação e utilização do potencial calorífico da escuma para geração de energia (MAGDZIARZ \& WILK, 2013). Entretanto, em ambos os casos, o resíduo sólido ao final do processo - torta, no caso da extração do óleo, ou cinzas, no caso do uso como combustível - necessita de disposição apropriada para a imobilização dos compostos inorgânicos. Assim, este trabalho teve como objetivo avaliar a utilização das cinzas da escuma como pigmento inorgânico, pois esse processo permite a imobilização das cinzas em uma matriz vítrea, visando à redução da possibilidade de contaminação ambiental, sendo uma alternativa de disposição final da escuma.

\section{METODOLOGIA}

\section{Obtenção e análise térmica da escuma}

As coletas de escuma foram realizadas sempre no mesmo reator, em três períodos diferentes durante o ano, com um intervalo médio de um mês 
entre cada coleta, tomando cuidado para que fossem realizadas entre os períodos de remoção do material pela equipe da estação de tratamento de esgoto (ETE), de modo que representassem, de fato, diferentes amostras. A coleta foi realizada em três diferentes pontos do reator: uma no centro dele e outras duas nas extremidades, sendo esses pontos diametralmente opostos. Tais pontos compõem uma única amostra com aproximadamente $2 \mathrm{~kg}$ de escuma, que foi depositada em superfície plana e quarteada. De cada fração foram coletadas alíquotas em torno de $25 \mathrm{~g}$, sendo transferidas para um frasco com tampa devidamente identificado, obtendo-se uma amostra de $100 \mathrm{~g}$ de escuma, utilizada para todas as análises. Esse procedimento foi realizado para as três coletas, visando obter amostras representativas. Nessa etapa de coleta, foram retirados os resíduos grosseiros, que consistiam basicamente de pequenos pedaços de plástico. Esses resíduos equivaliam, em massa, em torno de $0,5 \%$ da massa total coletada. As amostras obtidas após procedimento mencionado foram denominadas como mostrado no Quadro 1.

A decomposição térmica das amostras de escuma foi acompanhada por termogravimetria (TG) e análise térmica diferencial (DTA) (SDT 2960 Simultaneous DSC-TGA), com atmosfera de ar sintético - fluxo de $0,1 \mathrm{~L} \cdot \mathrm{min}^{-1}$, valor padrão para o equipamento utilizado - , taxa de aquecimento de $15^{\circ} \mathrm{C} \cdot \mathrm{min}^{-1}$ - esta taxa foi utilizada para uma análise rápida, sem prejuízo ao resultado final - e temperatura final de $900^{\circ} \mathrm{C}$. Foram utilizados cadinhos de alumina, e a massa para todas as amostras foi de $10 \mathrm{mg}$, valor limite devido às dimensões do cadinho utilizado.

\section{Obtenção das cinzas}

As amostras de escuma foram transferidas para cadinhos de porcelana de $50 \mathrm{~mL}$ e tratadas termicamente a $900^{\circ} \mathrm{C}$ - temperatura final da análise de TG, em que foi verificada que não havia mais perda de massa - em forno tipo mufla (HydroSan) durante $1 \mathrm{~h}-$ tempo mínimo suficiente para remoção total de matéria orgânica, determinado após realização de diversos ensaios - em atmosfera de ar estático e taxa de aquecimento de $10^{\circ} \mathrm{C} \cdot \mathrm{min}^{-1}$. Cada cadinho de porcelana continha aproximadamente $10 \mathrm{~g}$ de escuma, de modo que a calcinação fosse homogênea na superfície e no interior da amostra. As cinzas obtidas após o tratamento térmico foram desaglomeradas em peneiras de abertura de $45 \mu \mathrm{m}$. Essa abertura foi

Quadro1 - Códigos das amostras de escuma coletadas e das respectivas cinzas obtidas após o tratamento térmico a $900^{\circ} \mathrm{C}$ durante $1 \mathrm{~h}$ em forno mufla em atmosfera de ar estático.

\begin{tabular}{|c|c|c|} 
Coleta & $\begin{array}{c}\text { Código da amostra } \\
\text { de escuma coletada }\end{array}$ & $\begin{array}{c}\text { Código das amostras obtidas } \\
\text { após tratamento térmico }\end{array}$ \\
\hline 1 & EM13 & RM13 \\
\hline 2 & EJ13 & RJ13 \\
\hline 3 & EA13 & RA13 \\
\hline
\end{tabular}

escolhida para reter a areia grosseira presente na amostra e obter um material finamente dividido e adequado para aplicação como pigmentos, isto é, que se dispersa facilmente no meio de aplicação. As amostras foram, então, denominadas como apresentado no Quadro 1.

\section{Caracterização das cinzas}

As cinzas obtidas a $900^{\circ} \mathrm{C}$ foram caracterizadas por análise térmica - TG e DTA, nas mesmas condições utilizadas para a escuma, mas com temperatura final de $1.100^{\circ} \mathrm{C}$. Nesse caso, a maior temperatura final de análise foi escolhida baseando-se na temperatura comum de trabalho usada para esmaltação de peças cerâmicas $-800^{\circ}-$ $1.100^{\circ} \mathrm{C}$ - , para, assim, escolher mais adequadamente as condições para aplicação das amostras como pigmentos. Além disso, foram utilizadas as técnicas de fluorescência de raios X - FRX, Axios Advanced, PANalytical, standardless -; de difração de raios X - DRX, Shimadzu XRD-6000; Cu-K $\alpha, 3-100^{\circ}, 0,02^{\circ} . \mathrm{s}^{-1}$-; de microscopia eletrônica de varredura por efeito de campo - FE-MEV, TESCAN MIRA3 LM, com as amostras em pó depositadas sobre fita de carbono e metalizadas com uma liga ouro-paládio (Au-Pd) - ; e de espectroscopia de reflectância difusa na região do UV-Vis - DRS UV-Vis, Varian CARY 50, sonda Barrelino, 360-800 nm, iluminante CIE D65, ângulo de observador padrão de $10^{\circ}$, padrão branco de sulfato de bário $\left(\mathrm{BaSO}_{4}\right)$.

\section{Aplicação das cinzas como pigmentos cerâmicos}

A escolha do esmalte foi realizada com base na estabilidade térmica do pigmento, como será discutido adiante. Utilizou-se uma frita com composição majoritária de dióxido de silício $\left(\mathrm{SiO}_{2}\right)$ e óxido de potássio $\left(\mathrm{K}_{2} \mathrm{O}\right)$, que possui temperatura de aplicação de $850^{\circ} \mathrm{C}$. Essa frita foi misturada a caulim $-8 \%$ em massa. A esse material foram adicionados os aditivos carboximetilcelulose (CMC) - 0,15\% $\mathrm{m} / \mathrm{m}$ - e tripolifosfato de sódio (TPF) $-0,10 \% \mathrm{~m} / \mathrm{m}$. A mistura final foi moída por 24 horas em moinho de bolas - gira-moinhos de construção própria, utilizando jarro de porcelana Chiarotti, diâmetro de $150 \mathrm{~mm}$, capacidade de $1.000 \mathrm{~mL}$, com carga de $1.250 \mathrm{~g}$ de esferas de alumina com diâmetro médio de $10 \mathrm{~mm}$ e massa unitária média de 3,5 $\mathrm{g}$ - por via úmida com álcool isopropílico, sendo este utilizado para facilitar a posterior secagem do material. Por fim, o conteúdo moído foi seco em estufa (Brasdonto, modelo 4) a $70^{\circ} \mathrm{C}$ até a obtenção de uma massa constante e desaglomerada utilizando-se peneira com malha abertura de 325 Mesh (45 $\mu \mathrm{m})$. Nesse caso, a abertura foi escolhida para obter um material finamente dividido e homogêneo, que garantisse a esmaltação adequada, isto é, a fusão homogênea do esmalte durante o aquecimento. Assim, se obtém um esmalte brilhante, com características comparáveis aos comerciais. O material obtido ao fim do processo foi denominado 
esmalte base, cuja formulação objetivou melhorar as propriedades do esmalte pigmentado, reduzindo rachaduras por retração, bem como melhorar a sua dispersão em meio aquoso, formando um material mais homogêneo para aplicação.

O esmalte pigmentado para a aplicação na peça cerâmica foi preparado utilizando-se $95 \%(\mathrm{~m} / \mathrm{m})$ de esmalte base e $5 \%(\mathrm{~m} / \mathrm{m})$ de pigmento, sendo a mistura moída em moinho de bolas — frasco de polietileno de alta densidade de $60 \mathrm{~mm}$ de diâmetro, volume de $250 \mathrm{~mL}$, com carga de $150 \mathrm{~g}$ de esferas de zircônia estabilizada com ítria com $10 \mathrm{~mm}$ de diâmetro médio e massa unitária de $3,2 \mathrm{~g}$ - por via úmida com álcool isopropílico por $10 \mathrm{~min}$. Após a secagem, foram adicionados $50 \%(\mathrm{~m} / \mathrm{m})$ de água destilada. A aplicação foi realizada com um aplicador manual Binil (SERVITECH), com espessura de $0,5 \mathrm{~mm}$.

A esmaltação foi realizada em forno mufla a $850^{\circ} \mathrm{C}$, com taxa de aquecimento de $10^{\circ} \mathrm{C} \cdot \mathrm{min}^{-1}$ e tempo de patamar de $20 \mathrm{~min}$. Como pigmento padrão (PP), foi utilizado um pigmento comercial de cor alaranjado (COLORONDA S.L.; OB-112) com estrutura do tipo espinélio, composto por cromo $(\mathrm{Cr})$, ferro $(\mathrm{Fe})$, zinco $(\mathrm{Zn})$ e alumínio $(\mathrm{Al})$.

As cores dos pigmentos esmaltados foram caracterizadas por espectroscopia de reflectância difusa na região do UV-Vis nas mesmas condições descritas para os pigmentos em pó.

\section{RESULTADOS E DISCUSSÃO}

\section{Análise térmica da escuma}

As curvas obtidas na análise termogravimétrica (TG/DTA) se encontram na Figura 1 e os dados de perda de massa são descritos na Tabela 1.

A perda de massa que ocorre até a temperatura de aproximadamente $120^{\circ} \mathrm{C}$ foi associada à perda de umidade. A perda de massa em torno de $70 \%$ indica grande quantidade de água na amostra, o que é coerente com a ausência da etapa de secagem prévia das amostras. A essa temperatura também foi associada a perda de voláteis. Para avaliar essa informação, $10 \mathrm{~g}$ de escuma foram colocados em cadinho de porcelana e mantidos a $120^{\circ} \mathrm{C}$ durante $1 \mathrm{~h}$, no mesmo forno utilizado para o tratamento térmico da escuma. Passado esse tempo, verificou-se que o odor característico da escuma havia desaparecido.

Pela Figura 1A, fica evidente que a amostra EJ13 possui menor teor de umidade e voláteis que as demais, pois a perda de massa é inferior às demais.

A perda de massa que ocorre entre $150^{\circ}$ e $650^{\circ} \mathrm{C}$ foi associada à degradação térmica da matéria orgânica presente na amostra, uma vez que a escuma bruta é composta majoritariamente por compostos orgânicos. Como a escuma apresenta, dentre vários compostos, o lodo do reator, é possível sugerir que os eventos exotérmicos verificados possam ser associados àqueles identificados em degradação térmica de lodos de estações de tratamento de esgoto. Sendo assim, o evento exotérmico verificado entre $200^{\circ}$ e $300^{\circ} \mathrm{C}$ pode ser associado à decomposição de grupos carboxílicos, possivelmente de lipídios presentes. Já o evento exotérmico entre $400^{\circ}$ e $500^{\circ} \mathrm{C}$ pode ser associado à degradação térmica de compostos orgânicos mais complexos (FRANCIOSO et al., 2010; DE OLIVEIRA SILVA et al., 2012). A amostra EJ13, embora possua menor teor de umidade e voláteis, possui maior perda de massa nesse segundo intervalo de temperatura, sugerindo que é composta por mais matéria orgânica. A amostra EA13 apresenta alteração no evento exotérmico entre $400^{\circ}$ e $500^{\circ} \mathrm{C}$ - Figura $1 \mathrm{~B}-$, sendo muito menos intenso do que os das demais amostras, sugerindo composição com menor teor de compostos que se decompõem nesse intervalo de temperatura. Magdziarz e Werle (2014) também relataram esses intervalos de perda de massa na análise de TG de lodo de esgoto em fluxo de ar sintético, apenas com variações na primeira etapa de perda de massa, uma vez que a análise foi feita utilizando lodo desidratado (MAGDZIARZ \& WERLE, 2014).

Ao fim da análise, foi verificada a presença de um resíduo de coloração marrom-alaranjada, estável até a temperatura final de análise, uma vez que no intervalo de $650^{\circ}$ a $950^{\circ} \mathrm{C}$ não foram verificados eventos endotérmicos ou exotérmicos associados à transição de fase. Esse resultado é muito próximo ao obtido por Magdziarz e Wilk (2013) para lodo de esgoto, já que eles observaram que a amostra de lodo analisada não perdia massa em temperaturas acima de $650^{\circ} \mathrm{C}$ (MAGDZIARZ \& WILK, 2013). O teor médio de cinzas obtido para as amostras, em relação à escuma bruta — sem secagem foi de $8,83 \pm 1,83 \%$.

De acordo com os dados obtidos pela análise térmica, considerando $1.000 \mathrm{~kg}$ de escuma bruta — sem prévia secagem —, é possível obter $88,3 \mathrm{~kg}$ de cinzas brutas e $66,22 \mathrm{~kg}$ de pigmentos, uma vez que em torno de $75 \%$ da amostra possui granulometria abaixo de $45 \mathrm{~mm}$.

A partir dos valores obtidos por TG, é possível estimar o teor de cinzas para a escuma, caso ela seja desidratada antes do processo de calcinação. Considerando que nenhuma massa seria perdida antes de $120^{\circ} \mathrm{C}$, o valor médio de perda de massa seria de $15,49 \%$ - intervalo de $150-650^{\circ} \mathrm{C}$. Partindo de $1.000 \mathrm{~kg}$ de escuma seca, poderiam ser obtidos em torno de $845,10 \mathrm{~kg}$ de cinzas brutas e, após separação granulométrica em peneira, podem ser obtidos $633,8 \mathrm{~kg}$ de cinzas passíveis de serem aplicados como pigmentos.

A escuma é um material muito heterogêneo desde a sua composição propriamente dita. Ela também pode variar de acordo com o local em que é coletada, uma vez que depende da composição do esgoto que chega ao reator, da presença ou não de infiltrações na rede - a qual pode aumentar a quantidade de compostos inorgânicos presentes -, dentre outros fatores. Além disso, a quantidade produzida e removida relatada pelas estações de tratamento 

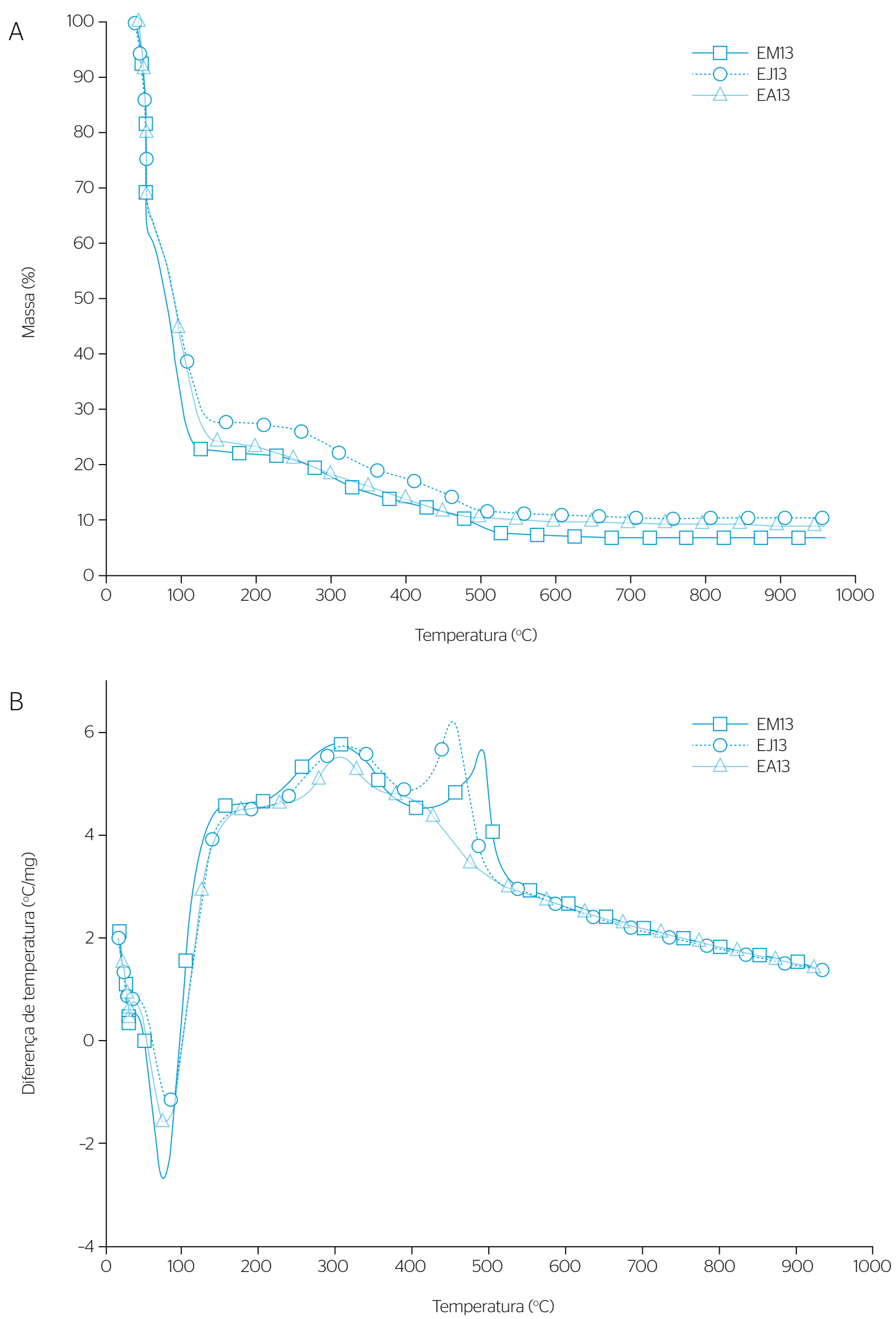

Figura 1 - Termogravimetria (A) e análise térmica diferencial (B) das amostras de escuma EM13 ( $\square$ ), EJ13 (O) e EA13 ( $\Delta$ ), obtidas em análise com fluxo de ar sintético, com aquecimento de $20^{\circ}$ a $900^{\circ} \mathrm{C}$. 
pode variar de maneira considerável, uma vez que é contabilizado o volume removido por caminhões de sucção e não em massa seca, dificultando a real estimativa dos dados. Entretanto, é possível verificar que os resultados obtidos no presente trabalho apresentam similaridades com diversos relatos da literatura sobre lodos de esgoto, que compõem parte da escuma utilizada, sendo um resultado bastante satisfatório.

\section{Caracterização das cinzas}

As curvas de TG e DTA das cinzas se encontram na Figura 2.

As amostras apresentam perfis de TG/DTA muito próximos, sugerindo que são muito parecidas entre si. Foram indicados no gráfico A, da Figura 2, três regiões. Na região (1), a $950^{\circ} \mathrm{C}$, é verificada a perda de massa pouco expressiva - inferior a $2 \%-$, sem associação a eventos endotérmicos ou exotérmicos, indicando estabilidade térmica das cinzas nesse intervalo de temperatura. Como exemplo de estabilidade térmica no caso de pigmentos, é possível mencionar o trabalho de Šulcová e Trojan (2008), que relatam que a perda de massa para o pigmento $\mathrm{Bi}_{1,2} \mathrm{Y}_{0,8} \mathrm{O}_{3}$ até $1.000^{\circ} \mathrm{C}$ é de $1 \%$. Assim, é possível dizer que o resultado obtido foi satisfatório, pois é próximo do obtido para um material sintético, muito mais homogêneo do que o obtido a partir da escuma (ŠULCOVÁ \& TROJAN, 2008). Além disso, verifica-se que essa é a região ideal para aplicação das amostras como pigmentos, pois não há perda de massa que possa ocasionar defeitos superficiais, como bolhas ou rachaduras. Com a escolha da temperatura, escolheu-se a frita com composição majoritária de $\mathrm{SiO}_{2}$ e $\mathrm{K}_{2} \mathrm{O}$, por apresentar temperatura de fusão em torno de $850^{\circ} \mathrm{C}$, ideal para a aplicação desejada.

$\mathrm{Na}$ região (2), a $1.000^{\circ} \mathrm{C}$, a perda de massa começa a extrapolar os $2 \%$, se intensificando em $1.100^{\circ} \mathrm{C}$; na região (3), por sua vez, a perda de massa chega a $6 \%$. Isso indica que, acima de $1.000^{\circ} \mathrm{C}$, alterações começam a ocorrer no sistema, possivelmente a decomposição de algum composto. Assim, a partir dessa temperatura, espera-se que as cinzas não apresentem bons resultados como pigmentos, por possível formação de bolhas no esmalte devido à perda de massa. Entretanto,

Tabela 1 - Variação de massa das amostras de escuma em diferentes intervalos de temperatura, observada na análise termogravimétrica em fluxo de ar sintético de 20 a $900^{\circ} \mathrm{C}$.

\begin{tabular}{l|c|c} 
Amostras & $\Delta \mathrm{m}$ & $\Delta \mathrm{m}$ \\
\hline EM13 & 77,79 & 150,11 \\
\hline EJ13 & 72,30 & 16,96 \\
\hline EA13 & 75,94 & 14,41
\end{tabular}

Variação de massa, em diferentes intervalos de temperatura (de 20 a $120^{\circ} \mathrm{C}$ e de $160-650^{\circ} \mathrm{C}$ ), observada na análise termogravimétrica das amostras de escuma de 20 a $900^{\circ} \mathrm{C}$ em fluxo de ar sintético. Acima de $650^{\circ} \mathrm{C}$ não se observa mais variação de massa para as amostras. para estimar o que ocorre no sistema é necessário conhecer a composição do mesmo. Na Tabela 2, são mostradas as composições das cinzas obtidas a $900^{\circ} \mathrm{C}$, determinadas por FRX, e as fases cristalinas, identificadas por DRX.

As fases cristalinas quartzo (dióxido de silício - $\mathrm{SiO}_{2}$ ), anidrita (sulfato de cálcio - $\mathrm{CaSO}_{4}$ ) e hematita (óxido de ferro $-\mathrm{Fe}_{2} \mathrm{O}_{3}$ ) também foram relatadas por Nowak, Aschenbrenner e Winter (2013) para cinzas de lodo de esgoto, além das fases de $\beta$-trifosfato de cálcio $\left(\mathrm{b}-\mathrm{Ca}_{3}\left(\mathrm{PO}_{4}\right)_{2}\right)$, sulfato de potássio e cálcio $\left(\mathrm{K}_{2} \mathrm{Ca}_{2}\left(\mathrm{SO}_{4}\right)_{3}\right)$, magnetita $\left(\mathrm{Fe}_{3} \mathrm{O}_{4}\right)$ e calcita $\left(\mathrm{CaCO}_{3}\right)$ (NOWAK et al., 2013).

Os resultados obtidos para a composição das cinzas da escuma são próximos aos obtidos por Magdziarz e Wilk (2013) para o lodo de ETE municipal, tendo esse como óxidos majoritários: dióxido de silício $\left(\mathrm{SiO}_{2}\right)$, óxido de cálcio $(\mathrm{CaO})$, óxido de magnésio $(\mathrm{MgO})$, óxido de ferro $\left(\mathrm{Fe}_{2} \mathrm{O}_{3}\right)$ e óxido de alumínio $\left(\mathrm{Al}_{2} \mathrm{O}_{3}\right)$ (MAGDZIARZ \& WILK, 2013). É importante destacar que, embora se tenham algumas variações nas quantidades, se compararmos as cinzas da escuma com o do lodo de ETE relatado, o $\mathrm{Fe}_{2} \mathrm{O}_{3}$ - cromóforo de interesse neste trabalho - apresenta um teor muito próximo, sendo 13,81\% relatado por Magdziarz e Wilk (2013), enquanto no presente trabalho tem-se em torno de $11,0 \%$. A vantagem da utilização do $\mathrm{Fe}^{3+}$ como cromóforo se deve a menor toxidade, se comparado aos elementos amplamente utilizados em pigmentos inorgânicos, como cromo $(\mathrm{Cr})$, cobalto $(\mathrm{Co})$, cádmio $(\mathrm{Cd}) \mathrm{e}$ chumbo (Pb) (OLEGÁRIO et al., 2013; 2014).

Assim como na análise termogravimétrica, verifica-se perda de massa a $1.050^{\circ} \mathrm{C}$ na análise por FRX. Isso pode ser associado à decomposição da anidrita impura, que ocorre de maneira geral no intervalo de 930 a $1.250^{\circ} \mathrm{C}$, mas em atmosfera oxidante (ar sintético) ocorre apenas acima de $1.000^{\circ} \mathrm{C}$, de acordo com a Equação 1 (O'GORMAN \& WALKER, 1973; VASSILEV et al., 1995; JIANGUO et al., 2007):

$\mathrm{CaSO}_{4(\mathrm{~s})} \stackrel{\Delta}{\rightarrow} \mathrm{CaO}_{(\mathrm{s})}+\mathrm{SO}_{3(\mathrm{~g})}$

Acima de $1.000^{\circ} \mathrm{C}$, quando há presença de sulfato de cálcio $\left(\mathrm{CaSO}_{4}\right)$, óxido de alumínio $\left(\mathrm{Al}_{2} \mathrm{O}_{3}\right)$ e dióxido de silício $\left(\mathrm{SiO}_{2}\right)$, pode ocorrer a formação de anortita $\left(\mathrm{CaAl}_{2} \mathrm{Si}_{2} \mathrm{O}_{8}\right)$ ou gehlenita $\left(\mathrm{Ca}_{2} \mathrm{Al}_{2} \mathrm{SiO}_{7}\right)$ (SUÁREZ-RUIZ \& CRELLING, 2008). A formação de tais compostos está possivelmente associada à reação no estado sólido dos compostos presentes, assim como à decomposição da anidrita $\left(\mathrm{CaSO}_{4}\right)$. Como na difração de raios $\mathrm{X}$, não foi identificada a presença de $\mathrm{Al}_{2} \mathrm{O}_{3}$ na forma de alumina. É possível que aluminossilicato de metais alcalinos e alcalinos terrosos - em especial de cálcio, uma vez que o mesmo se encontra entre os elementos majoritários - além do sistema $\mathrm{Al}_{2} \mathrm{O}_{3} .2 \mathrm{SiO}_{2}$, estejam presentes. Sendo assim, partindo-se do sistema $\mathrm{Al}_{2} \mathrm{O}_{3} .2 \mathrm{SiO}_{2}$, em presença do $\mathrm{CaO}$ a 

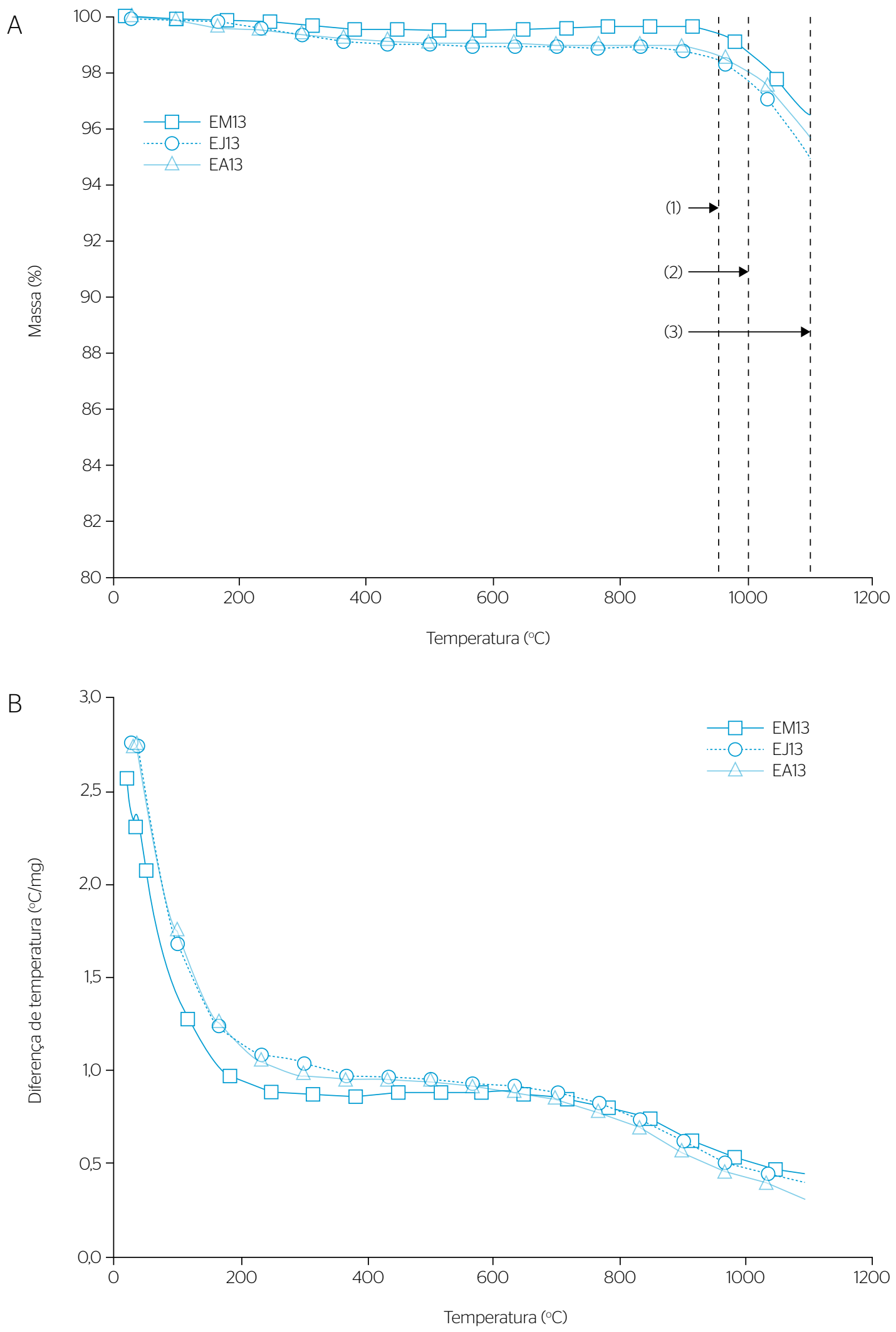

Figura 2 - Termogravimetria (A) e análise térmica diferencial (B) das amostras RM13 $(\square)$, RJ13 (O) e RA13 ( $\Delta$ ), obtidas em análise com fluxo de ar sintético, com aquecimento de 20 a $900^{\circ} \mathrm{C}$. 
partir da decomposição da anidrita, podemos escrever as Equações 2 e 3 (JIANGUO et al., 2007).

$\mathrm{Al}_{2} \mathrm{O}_{3} \cdot 2 \mathrm{SiO}_{2}+2 \mathrm{CaO} \rightarrow \mathrm{Ca}_{2} \mathrm{Al}_{2} \mathrm{SiO}_{7}+\mathrm{SiO}_{2}$ (Gehlenita)

$\mathrm{Al}_{2} \mathrm{O}_{3} \cdot 2 \mathrm{SiO}_{2}+\mathrm{CaO} \rightarrow \mathrm{CaAl}_{2} \mathrm{Si}_{2} \mathrm{O}_{8}$ (Anortita)

A partir da composição média das cinzas das escumas, é possível, por meio de um diagrama de fase ternário $\mathrm{CaO}-\mathrm{Al}_{2} \mathrm{O}_{3}-\mathrm{SiO}_{2}$, prever se ocorrerá a formação da anortita ou gehlenita. Para tanto, os teores médios de $\mathrm{SiO}_{2}, \mathrm{Al}_{2} \mathrm{O}_{3}$ e $\mathrm{CaO}$ descritos na Tabela 2 foram normalizados em 100,00\%, obtendo-se 51,33\% de $\mathrm{SiO}_{2}, 31,32 \%$ de $\mathrm{Al}_{2} \mathrm{O}_{3}$ e $17,35 \%$ de $\mathrm{CaO}$. A Figura 3 apresenta o diagrama de fase $\mathrm{CaO}-\mathrm{Al}_{2} \mathrm{O}_{3}$ $\mathrm{SiO}_{2}$, adaptado de Wu et al. (2010). O círculo preto indicado no diagrama de fase ternário indica a composição média normalizada das cinzas obtidas a $900^{\circ} \mathrm{C}$.

Para confirmar se ocorre a formação de anortita, seguindo a proposta do diagrama de fase, ou de gehlenita, as amostras obtidas a $900^{\circ} \mathrm{C}$

Tabela 2 - Resultado da análise química por Fluorescência de raios $X$ e principais fases identificadas na análise de Difração de raios $X$ das amostras RM13, RJ13 e RA13, mostrando os óxidos acima de 0,1\%.

\begin{tabular}{|c|c|c|c|c|}
\hline \multirow{2}{*}{ Óxidos } & \multicolumn{3}{|c|}{ Amostra } & \multirow{2}{*}{ Média } \\
\hline & RM13 & RJ13 & RA13 & \\
\hline $\mathrm{SiO}_{2}$ & 34,00 & 32,10 & 35,00 & $33,70 \pm 1,20$ \\
\hline $\mathrm{Al}_{2} \mathrm{O}_{3}$ & 21,30 & 18,80 & 21,60 & $20,57 \pm 1,26$ \\
\hline $\mathrm{CaO}$ & 13,30 & 12,00 & 8,88 & $11,39 \pm 1,86$ \\
\hline $\mathrm{Fe}_{2} \mathrm{O}_{3}$ & 11,20 & 9,60 & 10,90 & $10,57 \pm 0,69$ \\
\hline $\mathrm{SO}_{3}$ & 6,83 & 14,30 & 10,30 & $10,48 \pm 3,05$ \\
\hline $\mathrm{P}_{2} \mathrm{O}_{5}$ & 5,06 & 4,57 & 5,03 & $4,89 \pm 0,22$ \\
\hline $\mathrm{MgO}$ & 2,15 & 2,12 & 1,91 & $2,06 \pm 0,11$ \\
\hline $\mathrm{TiO}_{2}$ & 1,75 & 1,51 & 1,79 & $1,68 \pm 0,12$ \\
\hline $\mathrm{K}_{2} \mathrm{O}$ & 1,14 & 1,08 & 1,20 & $1,14 \pm 0,05$ \\
\hline $\mathrm{ZnO}$ & 0,27 & 0,22 & 0,19 & $0,27 \pm 0,03$ \\
\hline $\mathrm{BaO}$ & 0,17 & 0,12 & 0,16 & $0,15 \pm 0,02$ \\
\hline $\mathrm{Na}_{2} \mathrm{O}$ & 0,16 & 0,18 & 0,25 & $0,20 \pm 0,04$ \\
\hline $\mathrm{MnO}$ & 0,12 & 0,09 & 0,09 & $0,10 \pm 0,02$ \\
\hline$P F^{*}$ & 2,18 & 3,00 & 2,49 & $2,56 \pm 0,34$ \\
\hline
\end{tabular}

Fases principais identificadas por difração de raios $X \rightarrow$ quartzo $\left(\mathrm{SiO}_{2}\right)$, anidrita $\left(\mathrm{CaSO}_{4}\right)$, hematita $\left(\mathrm{Fe}_{2} \mathrm{O}_{3}\right)$

Foram representados apenas os óxidos presentes em média acima de 0,1\% em massa, pois abaixo desse valor os resultados passam a não ser tão confiáveis, pelo limite de detecção da análise. Óxidos representados com a composição mais estável. $\mathrm{SiO}_{2}$ : dióxido de silício; $\mathrm{Al}_{2} \mathrm{O}_{3}$ : Óxido de alumínio; $\mathrm{CaO}$ : óxido de cálcio; $\mathrm{Fe}_{2} \mathrm{O}_{3}$ : óxido de ferro; $\mathrm{SO}_{3}$ : trióxido de enxofre; $\mathrm{P}_{2} \mathrm{O}_{5}$ : pentóxido de fósforo; $\mathrm{MgO}$ : óxido de magnésio; $\mathrm{TiO}_{2}$ : óxido de titânio; $\mathrm{K}_{2} \mathrm{O}$ óxido de potássio; ZnO: óxido de zinco; $\mathrm{BaO}$ : óxido de bário: $\mathrm{Na}_{2} \mathrm{O}$ : óxido de sódio; $\mathrm{MnO}$ : óxido de manganês; PF*: Perda ao fogo quando aquecido a $1.050^{\circ} \mathrm{C} / 1 \mathrm{~h}$. foram submetidas a tratamento térmico em forno mufla Hydrosan, utilizando-se cadinhos de platina, a $1.100^{\circ} \mathrm{C}$ durante $1 \mathrm{~h}$. Os difratogramas obtidos são apresentados na Figura 4.

Verificou-se a presença de anortita $\left(\mathrm{CaAl}_{2} \mathrm{Si}_{2} \mathrm{O}_{8}\right)$, apresentando coerência com os dados previamente descritos. Também foi identificada a presença de hematita, $\mathrm{SiO}_{2}$ na forma de quartzo e $\mathrm{CaSO}_{4}$ na forma de anidrita, remanescente pelo pouco tempo de tratamento térmico.

A formação de outros compostos em temperaturas acima de $1.000^{\circ} \mathrm{C}$ é um fator limitante na temperatura de utilização das cinzas da escuma como pigmento sem que haja alteração em suas propriedades, corroborando os resultados da TG/DTA sobre a estabilidade térmica das cinzas.

Com relação à morfologia dos pigmentos, a Figura 5 apresenta as imagens obtidas por FE-MEV das cinzas com magnificação de 2.400 vezes.

É possível notar que as amostras apresentam partículas de diferentes formas e tamanhos. A partir de medidas do tamanho das partículas em diferentes micrografias de regiões distintas da amostra de cinza, pode-se inferir que as partículas apresentam tamanho médio no intervalo de 0,1 a $10,0 \mu \mathrm{m}$, faixa ideal para aplicação como pigmentos cerâmicos, uma vez que se dispersam bem no meio de aplicação sem serem solubilizados na matriz vítrea, o que leva à perda da cor (BONDIOLI; MANFREDINI; OLIVEIRA, 1998; MONRÓS et al., 2003).

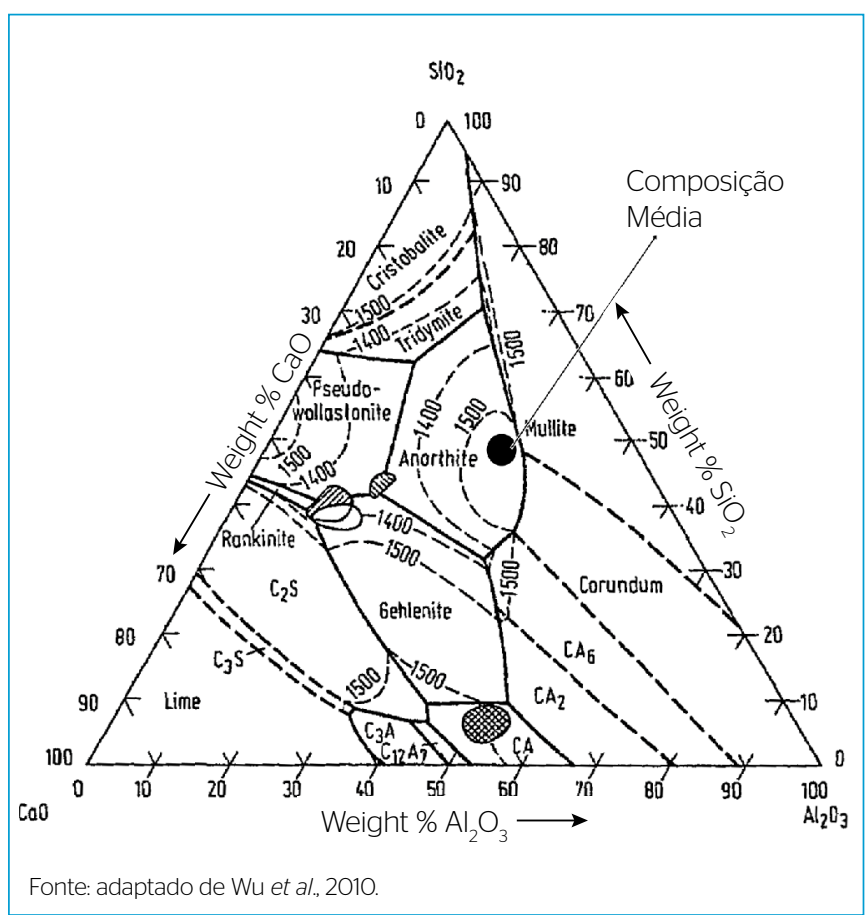

Figura 3 - Diagrama de fase ternário $\mathrm{CaO}-\mathrm{Al}_{2} \mathrm{O}_{3}-\mathrm{SiO}_{2}$, com a indicação da composição média das cinzas obtida por Fluorescência de raios $\mathrm{X}$. A marcação indica que, pela composição média, preferencialmente se forma anortita com o tratamento térmico das cinzas a $1.100^{\circ} \mathrm{C}$. 


\section{Aplicação das cinzas como pigmento inorgânico}

As amostras obtidas a $900^{\circ} \mathrm{C}$ e o pigmento padrão comercial (PP) foram aplicados na esmaltação de peças cerâmicas a $850^{\circ} \mathrm{C}$, temperatura utilizada em queimas decorativas. Essa temperatura foi escolhida tendo como base a análise de TG/DTA, como mencionado anteriormente. As coordenadas colorimétricas das amostras em pó e das respectivas peças esmaltadas se encontram na Tabela 3.

As cinzas apresentaram coordenadas colorimétricas $\mathrm{a}^{\star} \mathrm{e}^{\star}$ positivas, tendo, portanto, contribuição de vermelho e amarelo, respectivamente, levando à obtenção de pós com colorações alaranjadas. Os valores obtidos são próximos aos do pigmento padrão e entre si. Na esmaltação, obteve-se coloração intensa e opacidade, com o esmalte omitindo completamente a coloração do suporte cerâmico. Foi verificada grande variação de cor da peça esmaltada em relação à cor do pigmento em pó, devido à diminuição dos valores dos parâmetros $\mathrm{L}^{\star} \mathrm{e} \mathrm{b}^{\star}$. Com isso, foi possível identificar que as peças esmaltadas apresentaram a coloração mais escura - menor valor de $\mathrm{L}^{*}$ - e mais avermelhada - menor valor de $\mathrm{b}^{*}$ - que as respectivas amostras em pó. A variação da coloração com a esmaltação é algo comum, como pode ser observado para o pigmento comercial. Vale destacar que a variação de cor dos pigmentos obtidos a partir das cinzas, quando aplicados na esmaltação, foi muito menor que a observada para o pigmento comercial, sendo um ponto favorável, uma vez que o caráter heterogêneo do material de partida não ocasionou grandes problemas no resultado final.

Além da coloração obtida, a qualidade da esmaltação foi muito satisfatória. Foram obtidas peças cerâmicas com brilho, indicando que a granulometria dos pigmentos, de fato, era adequada para a aplicação. Mesmo quando se utiliza esmaltes brilhantes, na presença de pigmentos de granulometria menos controlada, a superfície do esmalte se torna fosca, pela alteração do espalhamento da luz ocasionada pela maior rugosidade da superfície. Outro fator importante que se destaca é a completa remoção da matéria orgânica com o tratamento térmico durante $1 \mathrm{~h}$. No caso de matéria orgânica residual, sua decomposição ocorreria durante o processo de esmaltação, levando à formação de bolhas no esmalte, decorrente da eliminação de gases. Pela superfície regular, verificou-se que o processo de obtenção das cinzas foi eficaz. Por fim, vale destacar

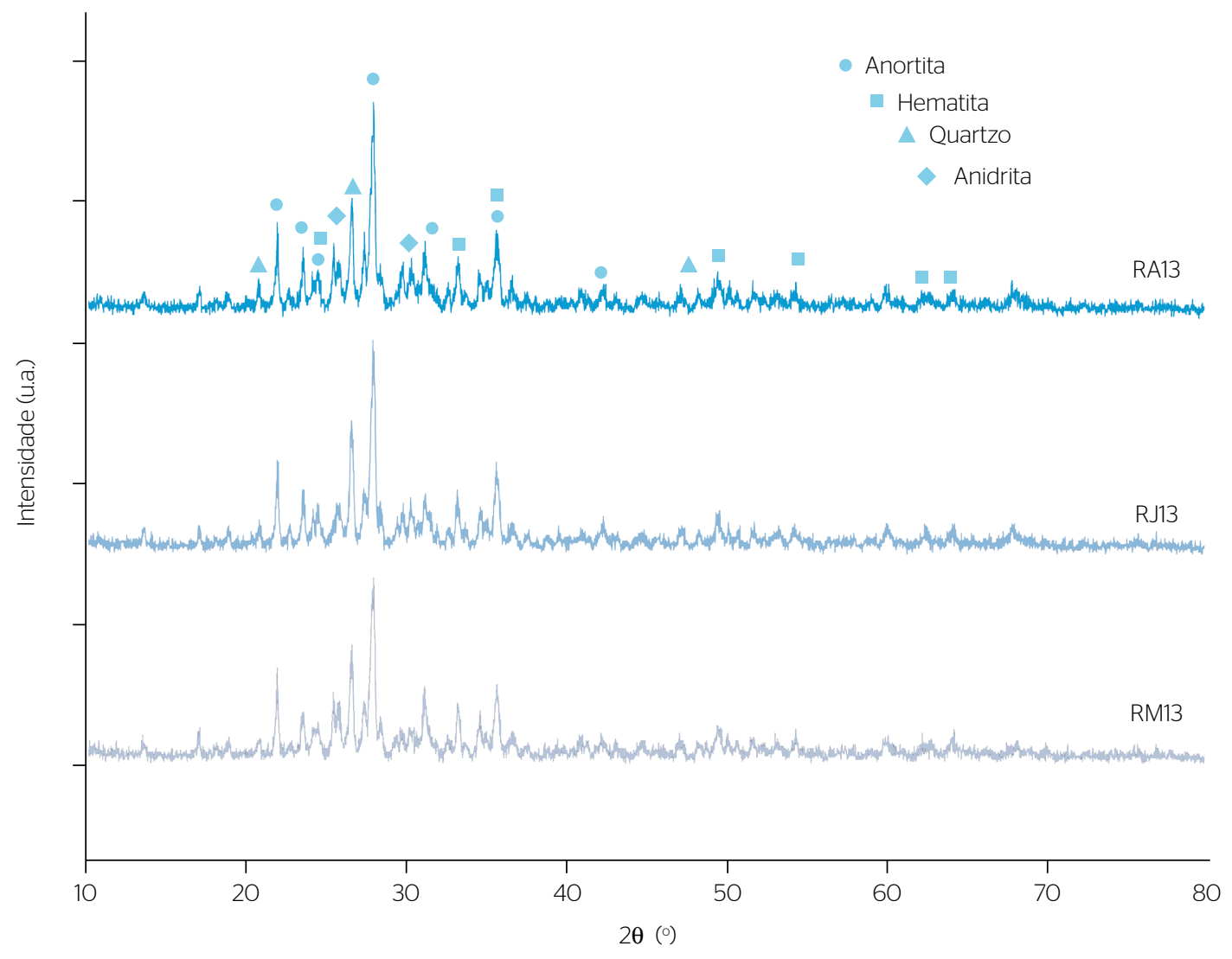

Figura 4 - Difratogramas de raios $X$ das cinzas tratadas termicamente $1.100^{\circ} \mathrm{C}$ durante $1 \mathrm{~h}$ em forno mufla com atmosfera de ar estático, com indicação das principais fases cristalinas identificadas. 


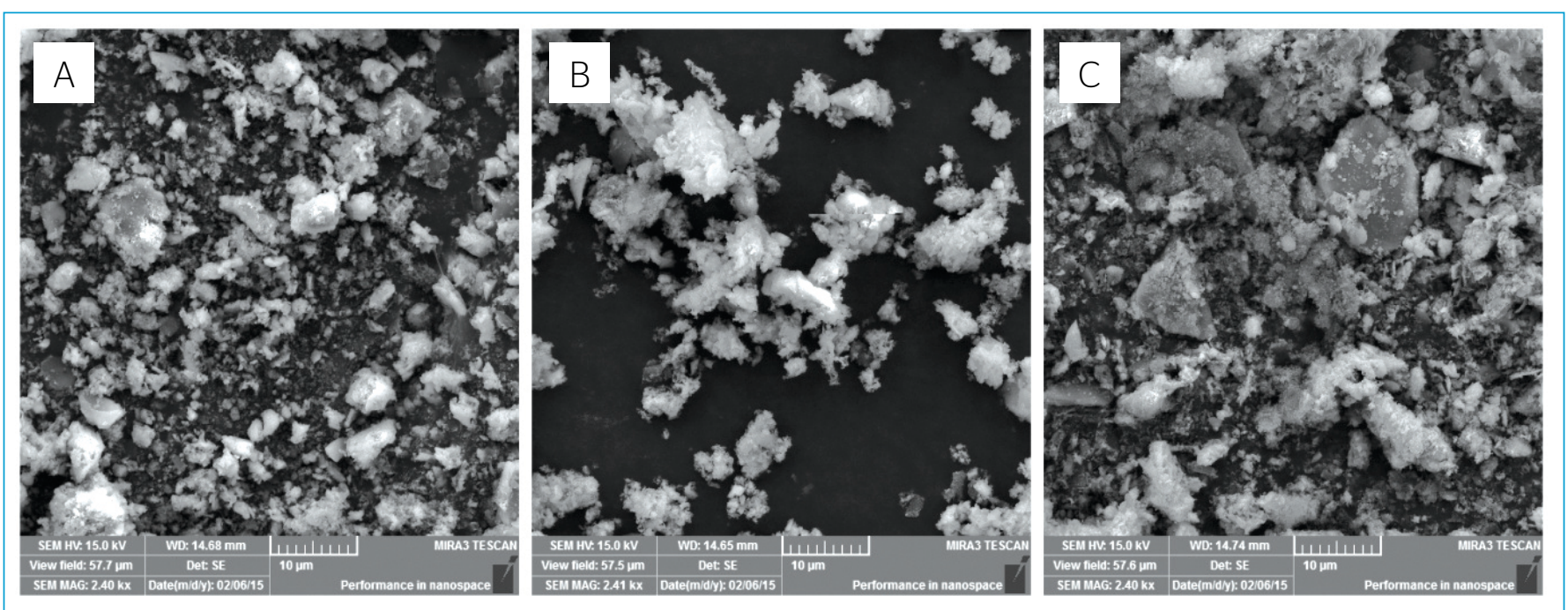

Figura 5 - Micrografias obtidas por Microscopia Eletrônica de Varredura por Efeito de Campo das cinzas RM13 (A), RJ13 (B) e RA13 (C), com magnificação de 2.400 vezes, com escala na imagem de $10 \mu \mathrm{m}$.

que a formulação do esmalte base atendeu às necessidades, visto que rachaduras decorrentes de retração do material não foram identificadas. Assim, fica evidente o potencial desse material para a aplicação proposta.

\section{CONCLUSÃO}

As cinzas das amostras de escumas apresentam propriedades interessantes, como a similaridade nas composições, as colorações intensas, a opacidade e a presença de elementos de baixa toxicidade, comuns no ambiente e no cotidiano. Além disso, as cinzas apresentam estabilidade frente à frita no intervalo de temperatura utilizado. Portanto, as amostras em pó obtidas são passíveis de serem aplicadas diretamente na esmaltação de peças cerâmicas nas temperaturas de queima decorativa, sendo uma possível aplicação para a fração inorgânica do resíduo gerado nos reatores anaeróbicos de tratamento de efluentes domésticos.
Tabela 3 - Coordenadas colorimétricas CIE L*a*b* das amostras RM13, RJ13 e RA13, do pigmento padrão (PP) em pó e das peças esmaltadas com os pigmentos. $\Delta \mathrm{E}_{00}$ é a variação de cor entre o pigmento em pó e a respectiva peça esmaltada.

\begin{tabular}{|c|c|c|c|c|c|c|c|c|}
\hline \multirow{3}{*}{ Amostra } & \multicolumn{6}{|c|}{ Coordenadas colorimétricas } & \multicolumn{2}{|c|}{ Variação de cor } \\
\hline & \multicolumn{3}{|c|}{ Amostras em pó } & \multicolumn{3}{|c|}{ Peças esmaltadas } & \multirow{2}{*}{$\Delta \mathrm{E}^{\mathrm{oo}}$} & \multirow{2}{*}{ Classificação* } \\
\hline & $\mathrm{L}^{*}$ & $\mathrm{a}^{*}$ & $\mathbf{b}^{*}$ & $\mathrm{~L}^{*}$ & $a^{*}$ & $b^{*}$ & & \\
\hline RM13 & 58,70 & 24,61 & 35,20 & 54,30 & 24,03 & 28,45 & 7,97 & $\begin{array}{l}\text { Facilmente } \\
\text { distinguível }\end{array}$ \\
\hline RJ13 & 62,38 & 21,88 & 31,38 & 52,58 & 17,14 & 21,35 & 10,10 & Muito grande \\
\hline RA13 & 56,72 & 22,51 & 32,17 & 47,16 & 21,42 & 24,68 & 10,16 & Muito grande \\
\hline PP & 64,77 & 19,67 & 29,26 & 44,10 & 21,42 & 24,51 & 20,03 & Muito grande \\
\hline
\end{tabular}

*Classificação de acordo com o relato de Silva, Petter e Schneider (2007).

\section{AGRADECIMENTOS}

Os autores agradecem às agências de fomento - CAPES, CNPq, Fundação Araucária -, ao C-LABMU/UEPG pelas análises realizadas, e ao técnico de ETE Josnei Souza.

\section{REFERÊNCIAS}

ANDREOLI, C.V. (2006) Biossólidos. In: ANDREOLI, C.V. Alternativas de uso de resíduos do saneamento. Rio de Janeiro: ABES. 417 p.

BARNETT, J.R.; MILLER, S.; PEARCE, E. (2006) Colour and art: a brief history of pigments. Optics \& Laser Technology, v. 38, n. 4-6, p. 445-453.

BONDIOLI, F.; ANDREOLA, F.; BARBIERI, L.; MANFREDINI, T.; FERRARI, A.M. (2007) Effect of rice husk ash (RHA) in the synthesis of $(\mathrm{Pr}, \mathrm{Zr}) \mathrm{SiO}_{4}$ ceramic pigment. Journal of the European Ceramic Society, v. 27, n. 12, p. 3483-3488.
BONDIOLI, F;; MANFREDINI, T.; OLIVEIRA, A.P.N. (1998) Pigmentos inorgânicos: projeto, produção e aplicação industrial. Cerâmica Industrial, v. 3, n. 4-6, p. 13-17.

BONDIOLI, F.; MANFREDINI, T.; SILIGARDI, C.; FERRARI, A.M. (2004) A new glass-ceramic red pigment. Journal of the European Ceramic Society, v. 24, n. 14, p. 3593-3601.

CHERNICHARO, C.A.L. (2010) Reatores anaeróbios. 2 ed. Minas Gerais: UFMG. 588 p. 
CHERNICHARO, C.A.L.; VAN LIER, J.B.; NOYOLA, A.; BRESSANI RIBEIRO, T. (2O15) Anaerobic sewage treatment: state of the art, constraints and challenges. Reviews in Environmental Science and Bio/Technology, v. 14, n. 4, p. 649-679.

COSTA, G.; DELLA, V.P.; RIBEIRO, M.J.; OLIVEIRA, A.P.N.; MONRÓS, G.; LABRINCHA, J. (2008) Synthesis of black ceramic pigments from secondary raw materials. Dyes and Pigments, v. 77, n. 1, p. 137-144.

DE OLIVEIRA SILVA, J.; RODRIGUES FILHO, G.; DA SILVA MEIRELES, C.; RIBEIRO, S.D.; VIEIRA, J.G.; DA SILVA, C.V.; CERQUEIRA, D.A. (2O12) Thermal analysis and FTIR studies of sewage sludge produced in treatment plants. The case of sludge in the city of Uberlândia-MG, Brazil. Thermochimica Acta, v. 528, n. 1, p. 72-75.

DI BITONTO, L.; LOPEZ, A.; MASCOLO, G.; MININNI, G.; PASTORE, C. (2016) Efficient solvent-less separation of lipids from municipal wet sewage scum and their sustainable conversion into biodiesel. Renewable Energy, v. 90, n. 1, p. 55-61.

FRANCIOSO, O.; RODRIGUEZ-ESTRADA, M.T;; MONTECCHIO, D.; SALOMONI, C.; CAPUTO, A.; PALENZONA, D. (2010) Chemical characterization of municipal wastewater sludges produced by two-phase anaerobic digestion for biogas production. Journal of Hazardous Materials, v. 175, n. 1-3, p. 740-746.

GEORGE, G.; SANDHYA KUMARI, L.; VISHNU, V.S.; ANANTHAKUMAR, S.; REDDY, M.L.P. (2008) Synthesis and characterization of environmentally benign calcium-doped $\mathrm{Pr}_{2} \mathrm{MO}_{2} \mathrm{O}_{9}$ pigments: applications in coloring of plastics. Journal of Solid State Chemistry, v. 181, n. 3, p. 487-492.

HABEEB, G.A. \& MAHMUD, H.B. (2010) Study on properties of rice husk ash and its use as cement replacement material. Materials Research, v. 13, n. 2, p. 185-190.

HAJJAJI, W.; ZANELLI, C.; SEABRA, M.P.; DONDI, M.; LABRINCHA, J.A. (2O11) $\mathrm{Cr}$-doped perovskite and rutile pigments derived from industrial by-products. Chemical Engineering Journal, v. 171, n. 3, p. 1178-1184.

HOSSEINI-ZORI, M. \& TAHERI-NASSAJ, E. (2011) Nano encapsulation of hematite into silica matrix as a red inclusion ceramic pigment. Journal of Alloys and Compounds, v. 510, n. 1, p. 83-86.

JANSEN, M. \& LETSCHERT, H. (2000) Inorganic yellow-red pigments without toxic metals. Nature, v. 404, n. 6781, p. 980-982.

JIANGUO, Y.; FURONG, D.; HONG, Z;; KEFA, C. (2007) Mineral conversion and microstructure change in the melting process of Shenmu coal ash. Asia-Pacific Journal of Chemical Engineering, v. 2, n. 3, p. 165-170.

MAGDZIARZ, A. \& WERLE, S. (2014) Analysis of the combustion and pyrolysis of dried sewage sludge by TGA and MS. Waste Management, v. 34, n. 1, p. 174-179.

MAGDZIARZ, A. \& WILK, M. (2013) Thermal characteristics of the combustion process of biomass and sewage sludge. Journal of Thermal Analysis and Calorimetry, v. 114, n. 2, p. 519-529.

MARANHA, F.G.; FIUZA, T.E.R.; SOUZA, E.C.F.; BORGES, J.F.M.; CUNHA, J.B.M.; ANDRADE, A.V.C.; ANTUNES, S.R.M.; ANTUNES, A.C.
(2016) Synthesis and characterization of pigments of the LaAl ${ }_{x} \mathrm{Fe}_{\mathrm{x}} \mathrm{O}_{3}$ system - application in ceramic and polymer. Dyes and Pigments, v. 133, n. 1, p. 304-310.

MARCELLO, R.R.; GALATO, S.; PETERSON, M.; RIELLA, H.G.; BERNARDIN, A.M. (2008) Inorganic pigments made from the recycling of coal mine drainage treatment sludge. Journal of Environmental Management, v. 88, n. 4, p. 1280-1284.

MARGUÍ, E.; IGLESIAS, M.; CAMPS, F.; SALA, L.; HIDALGO, M. (2016) Long-term use of biosolids as organic fertilizers in agricultural soils: potentially toxic elements occurrence and mobility. Environmental Science and Pollution Research, v. 23, n. 5, p. 4454-4464.

MELO, W.J.; MARQUES, M.O.; MELO, V.P.O. (2001) O uso agrícola do biossólido e as propriedades do solo. In: TSUTIYA, M.T.; ALÉM SOBRINHO, P.; HESPANHOL, I. Biossólidos na agricultura. São Paulo: SABESP. 468 p.

METCALF \& EDDY INC:; TCHOBANANOGLOUS, G.; BURTON, F.L.; STENSEL, H.D. (2003) Wastewater engineering: treatment and reuse. 4 ed. New York: McGraw-Hill. 1819 p.

MONRÓS, G.; BADENES, J.A.; GARCÍA, A.; TENA, M.Á. (2003) El color de la cerámica: nuevos mecanismos en pigmentos para los nuevos procesados de la industria cerámica. 3 ed. Castelló: Universitat Jaume I. 187 p.

NOWAK, B.; ASCHENBRENNER, P.; WINTER, F. (2013) Heavy metal removal from sewage sludge ash and municipal solid waste fly ash: a comparison. Fuel Processing Technology, v. 105, p. 195-201.

O'GORMAN, J.V. \& WALKER, P.L. (1973) Thermal behaviour of mineral fractions separated from selected American coals. Fuel, v. 52, n. 1, p. $71-79$

OLEGÁRIO, R.C.; SOUZA, E.C.F.; BORGES, J.F.M.; CUNHA, J.B.M.; ANDRADE, A.V.C.; ANTUNES, S.R.M.; ANTUNES, A.C. (2013) Synthesis and characterization of $\mathrm{Fe}^{3+}$ doped cerium-praseodymium oxide pigments. Dyes and Pigments, v. 97, n. 1, p. 113-117.

OLEGÁRIO, R.C.; SOUZA, E.C.F.; BORGES, J.F.M.; CUNHA, J.B.M.; ANDRADE, A.V.C:; ANTUNES, S.R.M.; ANTUNES, A.C. (2014) Synthesis and characterization of $\mathrm{CeO}_{2} \cdot \alpha-\mathrm{Fe}_{2} \mathrm{O}_{3}$ and $\mathrm{CeO}_{2} \cdot \mathrm{Pr}_{6} \mathrm{O}_{11}$ ceramic pigments through the solid state reaction and modified sol-gel method. Dyes and Pigments, v. 106, n. 1, p. 14-19.

OLIVEIRA, J.P.; ANTUNES, P.W.P.; PINOTTI, L.M.; CASSINI, S.T.A. (2O14) Physico-chemical characterization of oily sanitary waste and of oils and greases extracted for conversion into biofuels. Química Nova, v. 37, n. 4, p. 597-602.

POGGIANI, F. \& MULLER, H. (2005) Biossólido aumenta produtividade de eucaliptos. Visão Agrícola, v. 4, n. 1, p. 105-107.

RANKIN, R.S. \& SCHLENZ, H.E. (1947) Scum control in digesters. Sewage Works Journal, v. 19, n. 3, p. 405-414.

SAMAE POMERODE - Serviço Autônomo Municipal de Água e Esgoto de Pomerode. Estação de Tratamento de Esgoto (ETE). Disponível em: $<$ http://www.samaepomerode.com.br/index.php?pg=1078>. Acesso em: 26 jul. 2015. 
SANEPAR - Companhia de Saneamento do Paraná. Pesquisas. Disponível em: <http://site.sanepar.com.br/a-sanepar/inovacao/ pesquisas>. Acesso em: 18 jul. 2015.

SILVA, R.D.A.; PETTER, C.O.; SCHNEIDER, I.A.H. (2007) Avaliação da perda da coloração artificial de ágatas. REM: Revista Escola de Minas, v. 60, n. 3, p. 477-482.

SUÁREZ-RUIZ, I. \& CRELLING, J.C. (2008) Applied coal petrology: the role of petrology in coal utilization. USA: Academic Press. v. 40. 380 p.

ŠULCOVÁ, P. \& TROJAN, M. (2008) Thermal analysis of the $\left(\mathrm{Bi}_{2} \mathrm{O}_{3}\right)_{1-x}\left(\mathrm{Y}_{2} \mathrm{O}_{3}\right)_{x}$ pigments. Journal of Thermal Analysis and Calorimetry, v. 91, n. 1, p. 151-154.

TRANNIN, I.C.D.B.; SIQUEIRA, J.O.; MOREIRA, F.M.S. (2008) Atributos químicos e físicos de um solo tratado com biossólido industrial e cultivado com milho. Revista Brasileira de Engenharia Agrícola e Ambiental, v. 12, n. 3, p. 223-230.

VASSILEV, S.V.; KITANO, K.; TAKEDA, S.; TSURUE, T. (1995) Influence of mineral and chemical composition of coal ashes on their fusibility. Fuel Processing Technology, v. 45, n. 1, p. 27-51.

WU, X.; ZHANG, Z.; CHEN, Y.; ZHOU, T.; FAN, J.; PIAO, G.; KOBAYASHI, N.; MORI, S.; ITAYA, Y. (2O10) Main mineral melting behavior and mineral reaction mechanism at molecular level of blended coal ash under gasification condition. Fuel Processing Technology, v. 91, n. 11, p. 1591-1600.

ZHANG, Z.; ZHANG, L.; YIN, Y.; LIANG, X.; LI, A. (2O15) The recycling of incinerated sewage sludge ash as a raw material for $\mathrm{CaO}-\mathrm{Al}_{2} \mathrm{O}_{3}$ $\mathrm{SiO}_{2}-\mathrm{P}_{2} \mathrm{O}_{5}$ glass-ceramic production. Environmental Technology, v. 36, n. 9, p. 1098-1103. 